\title{
Non perturbative physics from NSPT: renormalons, the gluon condensate and all that
}

\section{Francesco Di Renzo*}

Dipartimento di Scienze Matematiche, Fisiche e Informatiche, Università di Parma and INFN,

Gruppo Collegato di Parma, I-43124 Parma, Italy

E-mail: francesco.direnzo@unipr.it

\section{Luigi Del Debbio, Gianluca Filaci}

Higgs Centre for Theoretical Physics, School of Physics \& Astronomy, University of Edinburgh, EH9 3FD, U.K

E-mail: luigi.del.debbioded.ac.uk, g.filacided.ac.uk

\begin{abstract}
Numerical Stochastic Perturbation Theory (NSPT) enables very high order computations in Lattice Gauge Theories. We report on the determination of the gluon condensate from lattice QCD measurements of the basic plaquette. This is a long standing problem, which was eventually solved a few years ago in pure gauge. In this context NSPT is crucial: it is actually the only tool enabling the subtraction of the power divergent contribution associated to the identity operator in the OPE for the plaquette. This subtraction is actually a delicate issue, since the perturbative expansion of the plaquette is on general ground expected to be an asymptotic one, due to renormalons. This in turn results in ambiguities and the separation of scales in the OPE does not correspond to a separation of perturbative and non-perturbative contributions. All in all, one needs to absorb the ambiguities attached to the perturbative series into the definition of the condensate itself, i.e. one needs a prescription. A possible one amounts to summing the perturbative series up to its minimal term, which means computing up to orders which only NSPT can aim at. Our computation is the first one in QCD, with massless staggered fermions. In order to remove the zero-mode of the gauge field, twisted boundary conditions are adopted for the latter, consistently coupled to fermions in the fundamental representation supplemented with smell degrees of freedom.
\end{abstract}

The 36th Annual International Symposium on Lattice Field Theory - LATTICE2018

22-28 July, 2018

Michigan State University, East Lansing, Michigan, USA.

\footnotetext{
* Speaker.
} 


\section{An old story: getting the gluon condensate from the OPE of the plaquette}

Non-perturbative effects in QCD often arise as power corrections. One tool to deal with them was put forward back in the late seventies by Shifman, Vainshtein and Zakharov and goes under the name of SVZ sum rules [1]. It amounts basically to an expansion of correlations functions in the vacuum condensates. One of the latter is the Gluon Condensate (GC)

$$
O_{G}=-\frac{2}{\beta_{0}} \frac{\beta(\alpha)}{\alpha} \sum_{a, \mu, v} G_{\mu \nu}^{a} G_{\mu \nu}^{a},
$$

which is defined in terms of the beta function $\beta(\alpha)=\frac{d \alpha}{d \ln \mu}=-2 \alpha\left[\beta_{0}(\alpha / 4 \pi)+\beta_{1}(\alpha / 4 \pi)^{2}+\ldots\right]$ and the field strength $G_{\mu \nu}^{a}$. In the following we will often relate the coupling $\alpha$ to the Wilson action coupling, i.e. $\alpha=N_{c} /(2 \pi \beta)$.

A non-perturbative determination of $O_{G}$ has been a longstanding challenge for lattice gauge theories. Naively there is a natural candidate to measure, i.e. the basic plaquette

$$
P=\frac{1}{6 N_{c} L^{4}} \sum_{\{P\}} \operatorname{Re}\left(\operatorname{Tr}\left(1-U_{P}\right)\right)
$$

where $U_{P}$ is the product of the link variables $U_{x \mu}$ around the $1 \times 1$ plaquette. Indeed in the naive continuum limit

$$
a^{-4} P \stackrel{a \rightarrow 0}{\longrightarrow} \frac{\pi^{2}}{12 N_{c}} O_{G}=\frac{\pi^{2}}{12 N_{c}}\left(\frac{\alpha}{\pi} G^{2}\right) \quad O_{G}=\frac{\alpha}{\pi} G^{2}[1+O(\alpha)],
$$

but then mixing with lower dimensional operators takes place (in the case at hand with the identity operator), which results in a power divergence

$$
a^{-4} P=a^{-4} Z(\beta) \mathbb{1}+\frac{\pi^{2}}{12 N_{c}} C_{G}(\beta) O_{G}+O\left(a^{2} \Lambda_{\mathrm{QCD}}^{6}\right)
$$

If one measures the plaquette by Monte Carlo one in turn has

$$
\langle P\rangle_{\mathrm{MC}}=Z(\beta)+\frac{\pi^{2}}{12 N_{c}} C_{G}(\beta) a^{4}\left\langle O_{G}\right\rangle+O\left(a^{6} \Lambda_{\mathrm{QCD}}^{6}\right),
$$

which can be read as an Operator Product Expansion (OPE): scales are separated and Wilson coefficients are computable in Perturbation Theory (PT)

$$
a^{-1} \gg \Lambda_{\mathrm{QCD}} \quad Z(\beta)=\sum_{n=0} p_{n} \beta^{-(n+1)}, \quad C_{G}(\beta)=1+\sum_{n=0} c_{n} \beta^{-(n+1)},
$$

in which $Z(\beta)$ is sometimes called the perturbative tail attached to the plaquette. (1.5) has been the starting point for a lattice determination of the gluon condensate. In principle the recipe is simple:

- compute the plaquette by Monte Carlo and in PT;

- subtract the latter from the former;

- repeat at different value for the coupling and look for asymptotic scaling; 


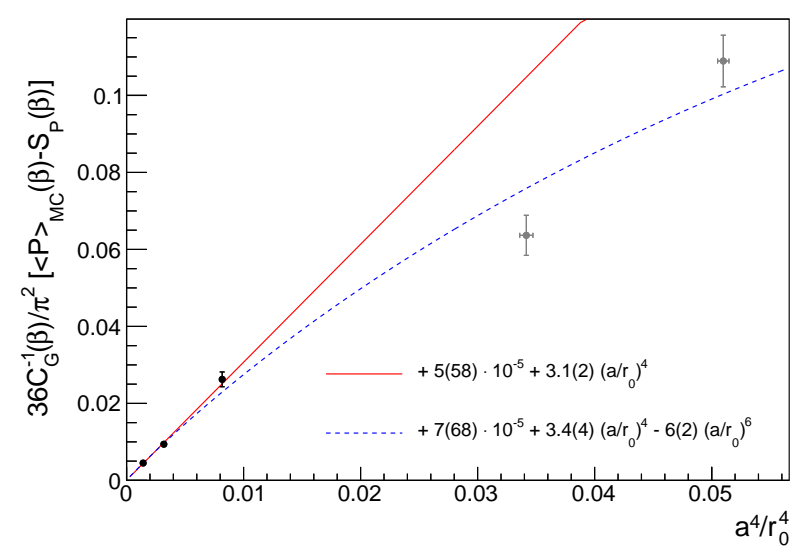

Figure 1: Scaling of the condensate with $a^{4}$ (solid red line, grey points excluded from the fit), with possibly a $a^{6}$ contribution (dashed blue line, grey points included in the fit).

- read the gluon condensate from the coefficient of the contribution scaling as $a^{4}$.

This approach goes back to the work of the Pisa group back in the late eighties and nineties [2]. Figure 1 shows the result of such a procedure in our computation, in which we considered $N_{f}=2$ QCD with (staggered) massless quarks [3]. While the procedure appears simple, there are quite a lot of steps to be taken in order to make sense out of it. All in all, the subtraction can make sense only in a given prescription and this ultimately has to do with the fact that the OPE separates the scales, but not perturbative and non-perturbative contributions. There is actually a contribution in the perturbative tail attached to the identity which scales exactly as the gluon condensate. This has to do with the asymptotic nature of perturbative series in field theories, which in turn gives rise to ambiguities which in asymptotically free field theories are known to have to do with the beta function: this is the famous story of IR renormalons.

\section{IR renormalons}

A dimension 4 condensate is expected to read

$$
W=\int_{0}^{Q^{2}} \frac{d k^{2}}{k^{2}}\left(\frac{k^{2}}{Q^{2}}\right)^{2} f(k)
$$

in which a UV cutoff $Q$ is in place and the form of the integrand is basically fixed by dimensional and renormalization group arguments: in particular $f(k)$ can be expressed in terms of the running coupling. If one picks the simplest expression $f(k)=\alpha(k)$ one is left with the expression

$$
W^{r e n}=\int_{0}^{Q^{2}} \frac{d k^{2}}{k^{2}}\left(\frac{k^{2}}{Q^{2}}\right)^{2} \alpha(k)
$$

A quick path into the emergence of (IR) renormalons goes through the change of variable

$$
z=z_{0}\left(1-\frac{\alpha(Q)}{\alpha(k)}\right) \quad z_{0} \equiv \frac{(4 \pi)^{2}}{3 \beta_{0}}
$$


which leaves us with

$$
W^{\text {ren }}=\mathscr{N} \int_{0}^{\infty} d z e^{-\beta z}\left(z-z_{0}\right)^{-1-\gamma}
$$

where $\gamma \equiv 2 \beta_{1} / \beta_{0}^{2}$. and a Borel integral can be recognized. In the previous expressions the beta function coefficients $\beta_{0}, \beta_{1}$ appear; we are retaining only these two terms in the expansion of the beta function, which is involved in the change of variable. (2.3) directly encodes the perturbative behaviour

$$
W^{r e n}=\sum_{l=1} \beta^{-l}\left[c_{l}^{r e n}+\mathscr{O}\left(e^{-z_{0} \beta}\right)\right] \quad c_{l}^{r e n}=\mathscr{N}^{\prime} \Gamma(l+\gamma) z_{0}^{-l} .
$$

All in all, we can say the following

- The coefficients of the expansion grow factorially.

- The integral has in turn a branch cut on the real axis and one needs a prescription to compute it. Typically this asks for a detour in the complex plane and as a result one picks up an imaginary part proportional to $e^{-\beta z_{0}}$.

- In order to sum the series one also needs a prescription, with an ambiguity which turns out to be just of the same order.

- The ambiguity we are left with scales just as the GC, i.e. as $e^{-\beta z_{0}} \sim \frac{\Lambda^{4}}{Q^{4}}$.

In view of all this, we need a prescription in order to perform the subtraction we are concerned with. But before getting into this, one question is in order: can we actually detect the divergent perturbative behaviour we are ready to take care of?

\section{Numerical Stochastic Perturbation Theory}

Explicitly inspecting an asymptotic behaviour asks for the computation of high orders. $\mathrm{Nu}-$ merical Stochastic Perturbation Theory $[4,5]$ (NSPT) is the numerical tool enabling such a computation. In its original formulation (the one we adhere to) the method amounts to the numerical implementation of Stochastic Perturbation Theory as originally introduced in the context of Stochastic Quantization [6]. For a given formulation of a lattice gauge theory (with gauge group $S U\left(N_{c}\right)$ ) based on an action $S[U]$, the link variable $U_{x \mu}$ evolves in the (fictitious) stochastic time $t$ according to the Langevin equation

$$
\frac{\partial}{\partial t} U_{x \mu}(t ; \eta)=\left(-i \nabla_{x \mu} S[U]-i \eta_{x \mu}(t)\right) U_{x \mu}(t ; \eta)
$$

where $\eta_{x \mu}$ is a gaussian noise, i.e. $\left\langle\eta^{a}(t)\right\rangle_{\eta}=0$ and $\left\langle\eta_{x \mu}^{a}(t) \eta_{y v}^{b}\left(t^{\prime}\right)\right\rangle_{\eta}=2 \delta^{a b} \delta_{v \mu} \delta_{y x} \delta\left(t-t^{\prime}\right)$. In our case we have in place both the gauge action $S_{G}[U]$ and the determinant of the Dirac operator $M[U]$, resulting in an effective action $S_{e f f}[U]=S_{G}[U]-\operatorname{Tr} \ln M[U]$. Asymptotically in the stochastic time, averages over the stochastic noise reconstruct the path integral averages one is interested in, i.e.

$$
\lim _{t \rightarrow \infty}\langle O[U(t ; \eta)]\rangle_{\eta}=\frac{1}{Z} \int D U e^{-S_{e f f}[U]} O[U] .
$$


NSPT is obtained by expanding the solution to (3.1) in powers of the coupling

$$
U_{x \mu}(t ; \eta)=1+\sum_{k=1} \beta^{-k / 2} U_{x \mu}^{(k)}(t ; \eta) .
$$

Plugging (3.3) into (3.1) results in a hierarchy of equations, exactly truncable at any given order. (3.2) holds in turn order-by-order and generates the perturbative expansions one is aiming at. We notice that explicitly one has

$$
\nabla_{x \mu} S=\sum_{a} T^{a} \nabla_{x \mu}^{a} S \quad \nabla_{x \mu}^{a} S_{e f f}=\nabla_{x \mu}^{a} S_{G}-\nabla_{x \mu}^{a} \operatorname{Tr} \ln M=\nabla_{x \mu}^{a} S_{G}-\operatorname{Tr}\left(\left(\nabla_{x \mu}^{a} M\right) M^{-1}\right)
$$

where the second (fermionic) contribution can be obtained via a stochastic estimator (multi-indices are in place)

$$
\operatorname{Re}\left(\xi_{k}^{\dagger}\left(\nabla_{x \mu}^{a} M\right)_{k l}\left(M^{-1}\right)_{l n} \xi_{n}\right)
$$

in which a second noise has been introduced satisfying $\left\langle\xi_{i} \xi_{j}\right\rangle_{\xi}=\delta_{i j}$. A numerical integration scheme is needed, e.g. Euler. This is not yet the end of the story, since a stochastic gauge fixing is needed to tame gauge modes $[4,5]$.

To avoid zero modes we use twisted boundary conditions (a choice first made in NSPT in [7])

$$
U_{\mu}(x+L \hat{v})=\Omega_{v} U_{\mu}(x) \Omega_{v}^{\dagger} \quad \Omega_{v} \Omega_{\mu}=z_{\mu \nu} \Omega_{\mu} \Omega_{v} \quad z_{\mu v} \in Z_{N_{c}}
$$

and consistently give fermions (in the fundamental representation) smell degrees of freedom, i.e. copies which transform into each other according to the anti-fundamental representation of the gauge group (physical observables are singlets)

$$
\psi(x+L \hat{v})_{i r}=\sum_{j, s}\left(\Omega_{v}\right)_{i j} \psi(x)_{j s}\left(\Omega_{v}^{\dagger}\right)_{s r} .
$$

After an exploratory study of critical mass for Wilson fermions, we eventually chose staggered fermions (for the first time introduced in NSPT); an obvious choice, given our aiming at high orders. In a preliminary phase we made use of an updated version of the PRLGT code [8], while production runs were performed in a new GRID [9] NSPT environment ${ }^{1}$.

Very high orders for toy models are known to display numerical instabilities [10] in NSPT. On the other side these had never been reported for gauge theories (not even for high orders) in the quenched case [11]. With fermions in place, we indeed found instabilities at high orders: see Figure 2. One should keep in mind that in NSPT physical observables do admit an asymptotic limit; still, we do not know that much on variances (which are not connected to physical observables). As expected on general grounds, the problems are milder on bigger volumes (see Figure 2). On one side, it is reassuring to notice that once a fluctuation takes place, the restoring force eventually takes the signal back around its average value; on the other side, this can be not the case if fluctuations are frequent enough to loose the signal.

\footnotetext{
${ }^{1}$ We thank P. Boyle and G. Cossu for their support.
} 

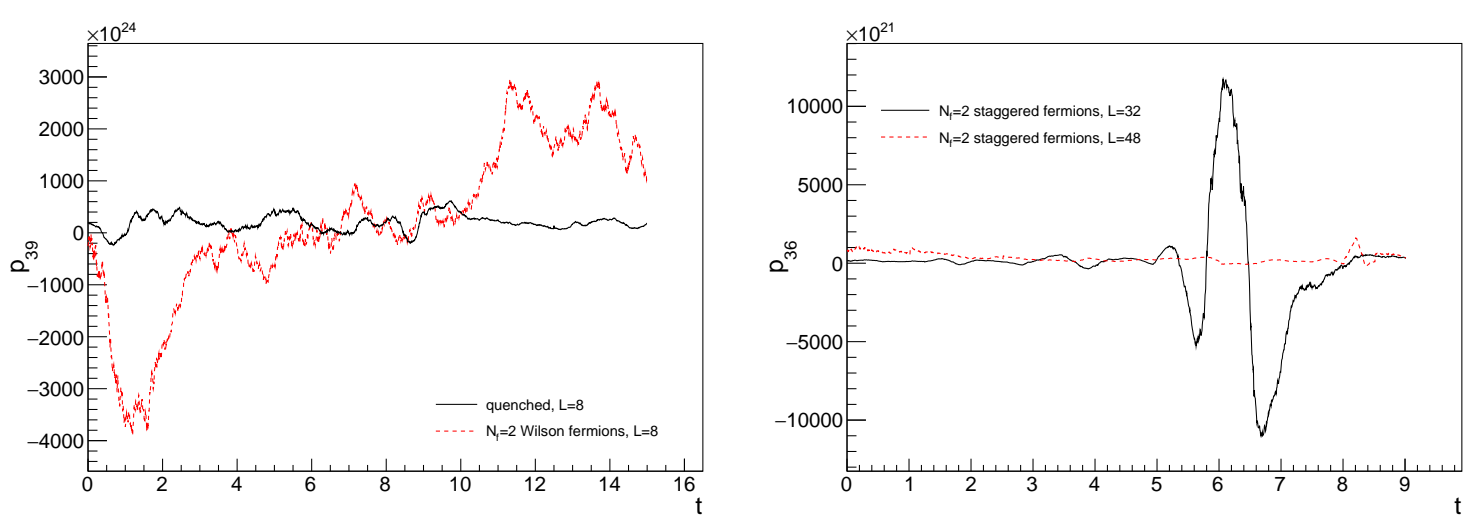

Figure 2: Examples of spikes at high orders. Left $\left(\beta^{-39}\right)$ : quenched vs unquenched (Wilson fermions); right $\left(\beta^{-36}\right)$ : $48^{4}$ vs $32^{4}$ (staggered fermions).

\section{Numerical results}

Detecting the renormalon growth has been a challenge for NSPT since the very early days of the method [12]. In recent years, the problem was successfully solved in the pure gauge case [11]. One needs to inspect the ratio

$$
\frac{p_{n}}{n p_{n-1}}=\frac{3 \beta_{0}}{16 \pi^{2}}\left[1+\frac{2 \beta_{1}}{\beta_{0}^{2}} \frac{1}{n}+O\left(\frac{1}{n^{2}}\right)\right], \quad \text { where } \quad\langle P\rangle_{\text {pert }}=\sum_{n=0}^{\infty} p_{n} \beta^{-(n+1)} .
$$

This is depicted in Figure 3. Within errors the asymptotic behaviour appears to show up, even if it is hard to get a definite answer: do the points really flatten to lay on the horizontal line (the signature of renormalons)? Our points cross the line, but finite size effects are there. A definite answer can only come from a careful analysis of finite size effects: the latter have already been studied in the literature $[13,11]$, a study that we do plan to repeat for the case at hand. Having said this, we do see an inversion point when we sum the series: although eventually regularised by the

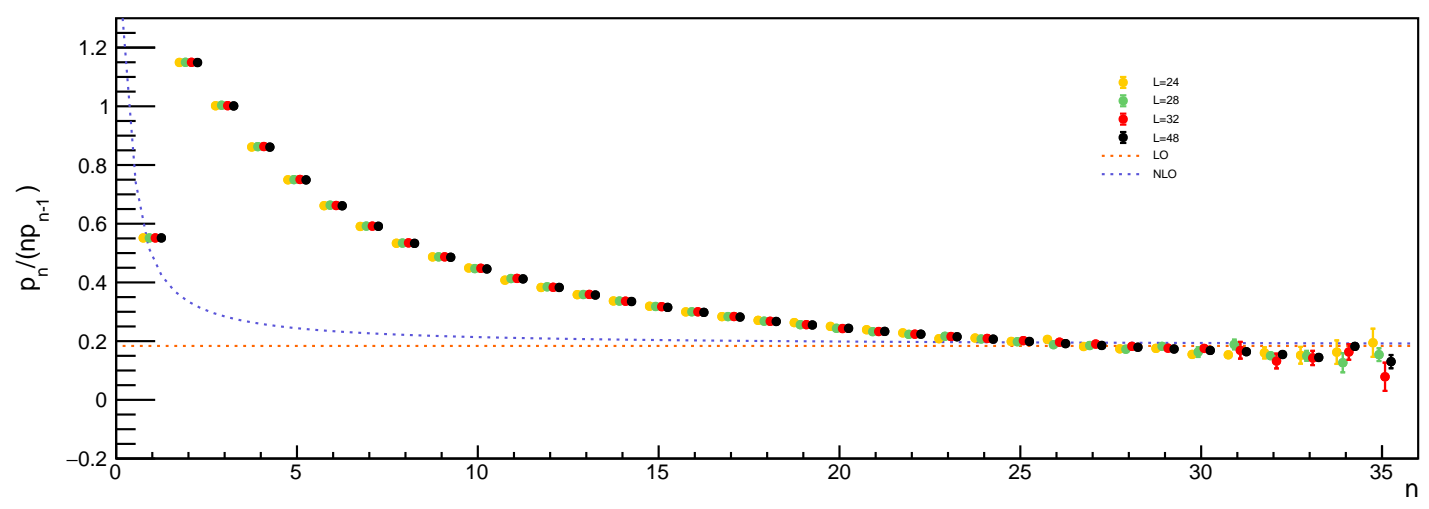

Figure 3: Ratio $p_{n} /\left(n p_{n-1}\right)$ extracted from our data at $L=24,28,32,48$ compared to leading order (LO) and next-to-leading order (NLO) corrections: see Eq (4.1). In order to be visible, points referring to different volumes are placed side by side. 
finite volume, the series still appears asymptotic at the orders we are taking into account.

Given the renormalon divergence, one needs a prescription to sum the series and a natural one is just summing up to the minimal term (inversion point), which occurs at a given order $\bar{n}$. The final recipe to look for the gluon condensate thus amounts to

$$
S(\beta)_{P}=\sum_{n=0}^{\bar{n}} p_{n} \beta^{-(n+1)} \quad\left\langle O_{G}\right\rangle=\frac{36}{\pi^{2}} C_{G}^{-1}(\beta) a^{-4}\left[\langle P\rangle_{\mathrm{MC}}(\beta)-S_{P}(\beta)\right] .
$$

Truncating the series is one of the possible prescriptions to sum a divergent series. An intrinsic ambiguity can be defined as the imaginary part of the Borel integrand. For the GC this results in

$$
\delta\left\langle O_{G}\right\rangle=\frac{36}{\pi^{2}} C_{G}^{-1}(\beta) a^{-4} \sqrt{\frac{\pi \bar{n}}{2}} p_{\bar{n}} \beta^{-\bar{n}-1}
$$

We stress that any prescription to sum the series amounts to having the ambiguity absorbed in the definition of the GC in the OPE. One should always bear this in mind when inspecting results like the one shown in Figure 1.

\section{References}

[1] M. Shifman, A. Vainshtein and V. Zakharov, QCD and Resonance Physics. Theoretical Foundations, Nucl. Phys., B147 (1979), 385.

[2] B. Allés, M. Campostrini, A. Feo and H. Panagopoulos, The three-loop lattice free energy, Phys. Lett., B324 (1994), 433 and references therein.

[3] L. Del Debbio, F. Di Renzo and G. Filaci, Large-order NSPT for lattice gauge theories with fermions: the plaquette in massless QCD, [1807.09518].

[4] F. Di Renzo, E. Onofri, G. Marchesini and P. Marenzoni, Four loop result in SU(3) lattice gauge theory by a stochastic method: Lattice correction to the condensate, Nucl. Phys., B426 (1994), 675.

[5] F. Di Renzo and L. Scorzato, Numerical stochastic perturbation theory for full QCD, JHEP, 10 (2004), 073.

[6] G. Parisi and Y.-S. Wu, Perturbation Theory Without Gauge Fixing, Sci. Sin., 24 (1981), 483.

[7] G. Bali, C. Bauer, A. Pineda and C. Torrero, Perturbative expansion of the energy of static sources at large orders in four-dimensional SU(3) gauge theory, Phys. Rev., D87 (2013), 094517.

[8] M. Brambilla, D. Dirk and F. Di Renzo, Code development (not only) for NSPT, in proceedings of the Lattice 2013 conference, PoS(LATTICE2013)418 (2014).

[9] P. Boyle, G. Cossu, A. Yamaguchi and A. Portelli, Grid: A next generation data parallel C++ QCD library, in proceedings of the Lattice 2015 conference, PoS(LATTICE2015)023 (2016).

[10] R. Alfieri, F. Di Renzo, E. Onofri and L. Scorzato, Understanding stochastic perturbation theory: Toy models and statistical analysis, Nucl. Phys., B578 (2000), 383.

[11] G. Bali, C. Bauer and A. Pineda, Perturbative expansion of the plaquette to $\mathscr{O}\left(\alpha^{35}\right)$ in four-dimensional SU(3) gauge theory, Phys. Rev., D89 (2014), 054505.

[12] F. Di Renzo, E. Onofri and G. Marchesini, Renormalons from eight loop expansion of the gluon condensate in lattice gauge theory, Nucl. Phys., B457 (1995), 202.

[13] F. Di Renzo and L. Scorzato, A Consistency check for renormalons in lattice gauge theory: $\beta^{-10}$ contributions to the SU(3) plaquette, JHEP, 10 (2001), 038. 